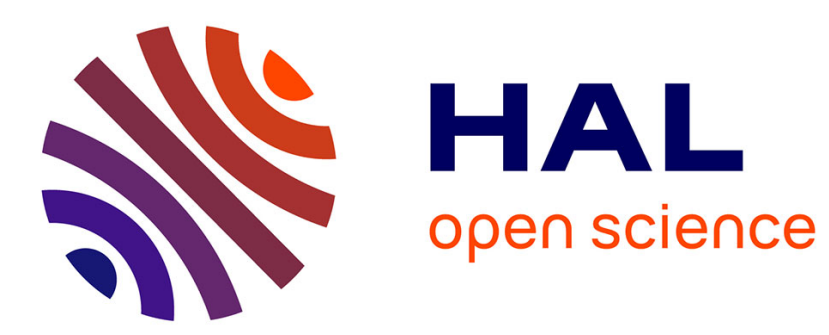

\title{
Insights from synthetic yeasts
}

Damien Coudreuse

\section{To cite this version:}

Damien Coudreuse. Insights from synthetic yeasts. Yeast, 2016, 33 (9), pp.483 - 492. 10.1002/yea.3169 . hal-01376255

\section{HAL Id: hal-01376255 https://hal-univ-rennes1.archives-ouvertes.fr/hal-01376255}

Submitted on 8 Nov 2016

HAL is a multi-disciplinary open access archive for the deposit and dissemination of scientific research documents, whether they are published or not. The documents may come from teaching and research institutions in France or abroad, or from public or private research centers.
L'archive ouverte pluridisciplinaire HAL, est destinée au dépôt et à la diffusion de documents scientifiques de niveau recherche, publiés ou non, émanant des établissements d'enseignement et de recherche français ou étrangers, des laboratoires publics ou privés. 
Insights from synthetic yeasts

\section{Damien Coudreuse}

SyntheCell team, Institute of Genetics and Development of Rennes, CNRS UMR 6290, 2 avenue du Pr. Léon Bernard, 35043 Rennes, France

\section{Abstract}

Synthetic biology is one of the most exciting strategies for the investigation of living organisms and lies at the intersection of biology and engineering. Originally developed in prokaryotes, the idea of deciphering biological phenomena through building artificial genetic circuits and studying their behaviors has rapidly demonstrated its potential in a broad range of fields in the life sciences. From the assembly of synthetic genomes to the generation of novel biological functions, yeast cells have imposed themselves as the most powerful eukaryotic model for this approach. However, we are only beginning to explore the possibilities of synthetic biology, and the perspectives it offers in a genetically amenable system such as yeast are endless.

\section{Graphical abstract}

(please use fig 1)

Synthetic biology has made a remarkable entry into the life sciences, playing an important role in our understanding of biological processes. From engineering novel genomes to transforming cells into chemical factories, yeasts represent ideal chassis for the application and future development of this bottom-up strategy in eukaryotes. 


\section{Introduction}

Complex genetic engineering in eukaryotic cells constitutes a cornerstone in the investigation of the functioning of living organisms. Pioneered by the introduction of simple, genetically amenable model systems, targeted genome alterations are integral to fundamental, translational and industrial biological research. In the past few years, the notion of synthetic biology has rapidly emerged to become a field of its own (Elowitz and Lim 2010), impacting virtually all domains in the life sciences. Referred to as a bottom-up approach, it focuses on the engineering of artificial molecular elements (Bashor et al. 2010; Nandagopal and Elowitz 2011). Thus, instead of classical top-down methods that involve perturbing existing processes in order to reveal how they are regulated, this strategy relies on building novel genetic networks and biological pathways to study their properties in vitro or in vivo. As will be described later, this encompasses systems that fulfill specific cellular functions as well as "functionless" circuits. However, what strictly defines synthetic biology remains a central point of discussion, and the boundary between standard genome editing and the study of synthetic networks is particularly difficult to establish. Nevertheless, beyond these semantic considerations, the logic of engineering artificial biological systems has undisputed value for both learning about natural functions as well as developing useful applications.

Despite the recent revolution in the editing of complex eukaryotic genomes (Mali, Esvelt and Church 2013; Doudna and Charpentier 2014; Sternberg and Doudna 2015), simple model systems remain the most appropriate chassis for synthetic studies. Initially focused on prokaryotic cells, the field of synthetic biology has logically expanded to the use of yeasts as central eukaryotic model organisms. Indeed, the robustness, growth characteristics, small genome size, ease of genetic manipulation and broad range of resources available for the most commonly used yeast species are particularly advantageous for advanced biological engineering. Furthermore, mathematical modeling and in silico analyses, which have proven to be a key aspect of a variety of synthetic biology projects (Wiechert 2002; Blazeck and Alper 2010; Osterlund, Nookaew and Nielsen 2012; Copeland et al. 2012), greatly benefit from the relative simplicity of the molecular mechanisms underlying cellular functions in yeast. Last but not least, the manipulation of yeast strains naturally 
raises fewer ethical issues than for higher eukaryotes.

Although in vitro approaches are playing important roles in the recent advances in synthetic biology, I will focus on in vivo studies in this review, considering both engineering at the molecular level as well as the exogenous control of cellular mechanisms. This does not constitute by any means an exhaustive overview of the exponentially growing use of synthetic biology in yeast, but rather aims at highlighting the different types of applications of this bottom-up strategy and discussing why they represent a step forward in fundamental and applied research.

\section{Genome engineering}

The conception of artificial genomes is the most prevalent application of synthetic biology in genetic model systems and was initially applied to viruses and bacterial species (Cello, Paul and Wimmer 2002; Pósfai et al. 2006; Gibson et al. 2010; Hutchison et al. 2016). In eukaryotes, engineering simplified genomes represents the ultimate approach for determining the minimal set of regulatory circuits and functions that are necessary and sufficient for sustaining cellular life. In particular, this provides an unprecedented platform for assessing intricate combinations of genes and associating them with complex phenotypes and behaviors. However, while this is one of the most anticipated outputs of such projects, artificial chromosomes are also likely to bring new insights to our understanding of genome structure. For the first time, fundamental questions such as the relevance of the specific ordering of genes and regulatory sequences along the chromosomes at the genome-wide scale will become accessible. We can also envisage addressing how and why genes are distributed among the various chromosomes of a species and the implications of having a particular chromosome count. Indeed, one could imagine producing strains with differing numbers of chromosomes but harboring the same initial set of information-carrying sequences. Finally, through the use of specific targeting tools, synthetic genomes will also constitute a unique way of investigating the relationship between the structure of the genome and its functional organization in the nuclear space. 
To date, the Saccharomyces cerevisiae Yeast Sc2.0 project remains the only comprehensive attempt to generate a synthetic eukaryotic genome (Dymond et al. 2011; Muller et al. 2012; Annaluru et al. 2014), and the assembly of the first complete artificial yeast chromosome synlll, related to chromosome III of $S$. cerevisiae, was reported in 2014 (Annaluru et al. 2014). By design, synIIl is a simplification of its parental chromosome in which a number of non-essential features have been deleted (e.g. transposons, introns, sub-telomeric repeats), while novel elements for further genome editing were introduced (LoxPSym sites, notably delineating non-essential genes). Beyond the proof-of-concept of the possibilities offered by modern synthesis techniques, this endeavor is poised to redefine the way we investigate the structure and function of eukaryotic genomes. From a different perspective, the built-in SCRaMbLE (Synthetic Chromosome Rearrangement and Modification by LoxP-mediated Evolution) system of Yeast Sc2.0 is an extremely powerful method to study the remodeling and evolvability of the genetic material as well as the costs/benefits of alternative genomes that may be compatible with growth (Dymond et al. 2011; Dymond and Boeke 2012; Shen et al. 2015). In addition, the assembly of artificial chromosomes in yeasts other than S. cerevisiae may have a significant impact on the investigation of particular processes. For instance, the study of RNA-mediated regulation, which has not been a critical aspect of the design strategy of Yeast Sc2.0, may benefit from the use of the fission yeast Schizosaccharomyces pombe, which is a widely-used model for this research area (Goto and Nakayama 2012; Holoch and Moazed 2015). Finally, in the context of metabolic engineering and cellular function hijacking, which will be discussed later, the idea of creating individual yeast genomes tailored to each specific project and need is unprecedented. Importantly, these are only few examples of the complete spectrum of groundbreaking possibilities that synthetic genomes will offer, and we are just beginning to uncover all of their potential. 
Designing novel genomes adapted to each question using the most appropriate testbed is the goal of whole genome engineering, but this nascent strategy remains a technical challenge: it took nearly five years to assemble synlll (Annaluru et al. 2014). Nevertheless, much can still be learned from smaller-scale synthetic approaches at the genome level in yeast. For instance, the generation of relatively healthy fission yeast strains with circular chromosomes allows the investigation of the functional and evolutionary relevance of operating with the endogenous linear chromosomes (Naito, Matsuura and Ishikawa 1998; Nakamura, Cooper and Cech 1998). Whether such studies belong to the field of synthetic biology to the same extent as artificial genome synthesis is a matter of appreciation. However, they apply the same logic of learning from the study of profound structural modifications of the genetic material. Therefore, crucial knowledge can already be acquired from focused genome manipulation before whole-genome synthesis becomes a routine technique.

\section{Hijacking molecular pathways}

A second highly-developed usage of synthetic biology is in the hijacking of cellular pathways and genetic networks for alternative artificial outputs. In particular, such approaches have been at the center of the field of metabolic engineering for the production of specific molecules of interest. Metabolic engineering was mostly initiated in prokaryotic models such as E. coli, converting micro-organisms into chemical factories to produce components as diverse as biofuels, biodegradable polymers or therapeutic molecules (Chen et al. 2013). Among eukaryotes, yeasts, and in particular $S$. cerevisiae, have rapidly become important vectors for in vivo chemical synthesis due to their ease of genetic manipulation, capacity to express and assemble complex enzymatic factories and robustness in challenging fermentation conditions (Ostergaard, Olsson and Nielsen 2000; Nevoigt 2008; Krivoruchko, Siewers and Nielsen 2011; Hong and Nielsen 2012). Among the most well-known products of metabolic engineering in yeast are the anti-malarial drug artemisinin (Paddon and Keasling 2014) and various alcoholic compounds that can serve as substitutes for fossil energies (e.g. ethanol, isobutanol) (Nielsen et al. 2013; Buijs, Siewers and Nielsen 2013). 
Metabolic engineering in yeast cells relies on the combination of three approaches, the importance of which varies depending on the target compound: 1) derivation of an endogenous biosynthetic cascade, 2) implementation of heterologous modules, and 3) tuning of the synthetic system for yield improvement. An early demonstration of the success of this methodology in assembling novel biosynthetic routes in yeast was demonstrated by the generation of a budding yeast strain capable of autonomously producing hydrocortisone, a major steroid used in the synthesis of a range of therapeutic drugs (Szczebara et al. 2003). This involved the rerouting of the ergosterol pathway, the integration of no less than eight mammalian and one plant genes in the system, and offered an alternative to the traditional, complex and expensive process of hydrocortisone synthesis. In addition, computational tools and mathematical modeling have played key roles in guiding the design and optimization of cellular factories in order to increase synthesis efficiency (Wiechert 2002; Blazeck and Alper 2010; Osterlund, Nookaew and Nielsen 2012; Copeland et al. 2012). In the case of artemisinin, the endogenous S. cerevisiae mevalonate pathway was rewired at the farnesyl pyrophosphate (FPP) stage: specific alterations in the expression of key factors of this cascade resulted in increased FPP production while reducing its use for sterol synthesis (Ro et al. 2006). FPP was then converted to amorphadiene and subsequently to artemisinic acid, the precursor of artemisinin, by introduction of the amorphadiene synthase gene and cytochrome P450 (together with its redox partner) from A. annua (Ro et al. 2006). This network allowed for the first efficient production of artemisinic acid from yeast cells. Variations on the backbone of this system, from tuning gene expression levels to performing codon optimization of the heterologous modules, improved the yield of this synthetic cascade, a critical parameter for the efficient and cost-effective production of therapeutic compounds (Paddon et al. 2013). The synthesis of biofuel candidates has followed the same rationale, initially focusing on reducing glycerol synthesis in favor of ethanol production. This required changes to the associated endogenous biosynthetic pathways at both the molecular and spatial levels as well as integration of heterologous modules (Buijs, Siewers and Nielsen 2013; Rabinovitch-Deere et al. 2013; Kricka, Fitzpatrick and Bond 2014). Finally, a recent extreme example is the assembly and optimization of a complete opioids synthesis pathway, involving the expression of more than twenty different enzyme activities from various organisms in a S. cerevisiae host (Galanie et al. 2015). This represents 
a first step towards a reduced dependency on farming for the production of these therapeutic molecules.

Beyond these very successful applications, hijacking endogenous functions through synthetic manipulation for the production of a growing range of molecules has become an intensive field of research and development in the biotechnology and pharmaceutical industries (Hong and Nielsen 2012; Borodina and Nielsen 2014). The concomitant efforts of the synthetic biology community to systematically develop new generic biological modules, from core components for circuit building (e.g. promoters, translational units) to composite networks with specific functions (e.g. genetic logic gates), are critical for the expansion of this domain. While S. cerevisiae remains the most widely used yeast in metabolic engineering, there has been an increasing interest in other yeast species with metabolic or growth properties that may be more appropriate for the production of specific compounds (Gellissen et al. 2005; Fitzpatrick et al. 2014). In this respect, the applications of yeast engineering inevitably intersect with fundamental research for the understanding of natural biological systems. Revealing differences in the tolerance of various yeast models to modulation of their metabolic pathways may provide new insights into the conditions in which they may have evolved.

\section{Recapitulating cellular functions}

Synthetic biology is also beginning to have an impact on fundamental research through the recapitulation of natural cellular functions. This is achieved by constructing simple artificial regulatory circuits that significantly differ from those normally operating in wild type cells while still regulating the same processes. Obviously, the engineering of such networks does not benefit from the same degree of freedom as that of in vitro strategies. Indeed, as the goal is to replace endogenous control systems by their minimal counterparts in living cells, the synthetic circuits must still possess some of the natural activities. This is particularly true for essential functions: for instance, if the activation of a signaling cascade results in the expression of an essential gene, the output of the engineered system is likely to require the eventual induction of the same gene. The host of genetic tools available 
in yeast is particularly adapted in this context as it is possible to construct inducible switches between endogenous and artificial pathways, an important feature when the functionality of a synthetic circuit cannot be predicted. So what do we learn from simply recreating normal functions with alternative networks? The potential of bottom-up strategies for understanding the foundations of cellular processes is immense, and once again, yeast cells have been at the forefront of these investigations in eukaryotes.

First, this represents a unique approach to go beyond the complexity of natural circuits and reveal the core principles underlying biological phenomena. Replacing a regulatory pathway by a simple artificial system for which we have a clear understanding of the inputs and outputs is powerful: it can identify the events that are necessary and sufficient for the associated function as well as the principles of its regulation. This has proven to be applicable for some of the most basic processes in eukaryotic cells, such as cell proliferation. In fission yeast, cell cycle progression is regulated by a complex and conserved network involving cyclin-dependent kinases (CDK) and various regulatory subunits of the cyclin family (Morgan 1997; 2007). Interestingly, the construction of a simple synthetic module consisting of a fusion of a single cyclin (cyclin B/Cdc13) with the fission yeast cell cycle CDK Cdc2 challenged the established model of cell cycle regulation (Fisher and Nurse 1996; Morgan 1997; 2007; Coudreuse and Nurse 2010). By design, this synthetic system bypasses a number of features believed to be critical for the cell cycle network, including the specific synthesis and degradation programs of the different factors, the requirement for distinct cyclin/CDK combinations, and the attainment of critical cyclin/CDK concentration ratios. Strikingly, cells operating solely with this minimal circuit showed normal cell cycle progression. This study led to the emergence of a modular model for the control of cell proliferation, in which the different phases of the cell cycle are not inherently organized in a directional cycle: cells simply respond to the CDK activity level to which they are exposed, regardless of their cycle stage (Coudreuse and Nurse 2010). The coupling of mathematical modeling of this simplified network with experimental approaches testing model predictions also allowed for a better understanding of the phenomenon of mitotic catastrophe, a lethal situation in which mitosis occurs prior to the completion of genome duplication (Gérard et al. 2015). 
Another example that exploits synthetic biology and modeling is the study of singularity in symmetry breaking in the budding yeast (Howell et al. 2009). Polarization in S. cerevisiae was proposed to rely on the assembly of polarity poles containing the conserved Rho GTPase Cdc42p through a diffusion-mediated positive feedback loop (Kozubowski et al. 2008; Goryachev and Pokhilko 2008). In order to assess the role of this amplification in the singularity of polarization, a synthetic system was generated in which an alternative actin-mediated feedback loop was engineered by fusing the Cdc42p-binding scaffold Bem1p (that promotes actin cable attachment via binding to Cdc42p) to the yeast exocytic v-SNARE Snc2p (that allows traffic on secretory vesicles and slow diffusion at the plasma membrane) (Howell et al. 2009). This system sustained polarization, although an increase in the occurrence of cells with two buds was observed. Modeling these observations led to the demonstration that slower competition between polarity foci in rewired cells was responsible for this abnormal phenotype and established that singularity in bud formation lies in the fast kinetics of this competition set by the diffusion-mediated amplification loop (Howell et al. 2009). These studies highlight how the combination of in vivo synthetic systems with mathematical modeling represents a powerful pipeline for deciphering normal cellular processes. In addition, the host of genetic tools available in yeast is particularly adapted for assessing complex essential functions: it is possible to construct inducible switches between endogenous and artificial pathways, an important feature when the functionality of a synthetic circuit cannot be predicted.

Second, while traditional top-down approaches have allowed us to describe the complexity of regulatory networks, the rationale for evolving a given circuit topology cannot be easily investigated using these strategies. Again, synthetic biology provides ways to answer these novel questions through assessing the costs/benefits of alternative architectures that may appear to efficiently recapitulate natural functions. For instance, this logic was applied in prokaryotes, where switching the topology of the feedback loop regulating the competence phase in Bacillus subtilis (from inhibition of an activator to activation of an inhibitor) resulted in reduction of the noise inherent to the endogenous system (Cağatay et al. 2009). However, this increased precision was accompanied by a reduction in the efficiency of DNA uptake for a wide range of DNA concentrations, which can be detrimental for adaptation in 
changing growing conditions (Cağatay et al. 2009). In yeast cells, while synthetic pathways recapitulating endogenous functions with alternative topologies are being generated (Takahashi and Pryciak 2008; Coudreuse and Nurse 2010; Tao, Calvert and Balasubramanian 2014), we are only in the initial stages of exploiting this approach. Assessing the long-term effects (e.g. genome stability, fitness) of artificially simplifying cellular machineries is therefore a promising avenue of research, and it is poised to bring novel insights into determinant factors of the evolution of eukaryotic circuits as we know them. In addition, the variety of yeast species that have been sequenced and genetically characterized will make it possible to compare the response of different cells to similar synthetic circuits, probing the robustness and plasticity of different yeasts. Finally, synthetic networks fulfilling essential functions represent unique tools for investigating the evolvability of regulatory pathways as a function of their topologies. In this context, the use of experimental evolution with engineered yeast strains will be key for learning more about evolutionary principles.

\section{From proof-of-concept circuits to novel programs and functions}

A number of pioneering studies in the early days of synthetic biology were conducted in bacteria and aimed at generating artificial circuits in vivo that did not carry any biological function (Sprinzak and Elowitz 2005). The goal was to put in a cellular context simple synthetic networks in order to describe and understand their operation and properties at a theoretical level. A well-known example of this is the investigation of synthetic oscillators, which provided a novel view of this common natural behavior (Elowitz and Leibler 2000; Stricker et al. 2008). In contrast with the different aspects of synthetic biology described above, implementing "functionless" autonomous systems requires minimizing their interactions with the biology of the host cell. This is clearly one of the areas that benefits the most from the use of mathematical models and their capacity to predict network behavior. Importantly, this approach can reveal conserved design principles in genetic networks, such as the essential inputs or appropriate dynamic properties for increased robustness (Mukherji and van Oudenaarden 2009). 
In eukaryotes, the engineering of genetic modules that have no biological function has not reached the sophistication of studies in prokaryotes, despite their recognized potential to identify the core features underlying cellular functions. However, the exponential development of new tools and synthetic bricks for building artificial systems, in particular in yeast, should provide the required versatility and flexibility. An interesting example from the budding yeast involves the rational design of a synthetic memory circuit that can trigger a switch between two stable states following a transient stimulation and subsequently maintain the activated state throughout several generations (Ajo-Franklin et al. 2007). This system consists of two simple components: 1) a transcriptional activator module, whose expression is induced in response to galactose, and 2) a reporter module, whose expression depends on the binding of the first module to its promoter. The reporter integrates an autofeedback loop, as it binds to the same regulatory elements as the activator and is fused to a transactivation domain, thereby promoting its own expression. Modeling of this system coupled to single-cell microscopy demonstrated that this simple network can only produce robust memory when a number of quantitative parameters are met, including the relative rates of production and decay of the activator and reporter as well as the cellular growth rate (Ajo-Franklin et al. 2007). For instance, memory in fast growing conditions required an increase in the strength of the autofeedback. While this purely synthetic system is unlikely to reflect the generation of complex memory in molecular pathways, it provides a minimal motif and a set of quantitative parameters that may help identify cellular functions with memory, such as cell fate decisions.

In yeast cells, a stronger focus has been placed on methods for reprogramming cellular physiology and on the generation of completely novel functions. For instance, the possibility to rationally redesign the expression landscape of the yeast genome by imposing synthetic transcriptional programs at various scales is an unprecedented approach for investigating the relationship between cellular behaviors (e.g. metabolic responses, control of cell fate, stress responses) and complex patterns of gene expression. With this in mind, synthetic long non-coding RNAs (IncRNAs) were constructed as powerful scaffolding platforms to specifically reprogram gene expression (Zalatan et al. 2015). This was achieved through extending the CRISPR single-guide RNA, which provides locus specificity, with additional motifs 
allowing the recruitment of RNA-binding proteins fused to either transcriptional activators or repressors. Thus, each Inc-RNA carries information for both the target gene and the pre-defined type of regulation. In principle, in the presence of dCas9, a given transcriptional program can therefore be imposed by simply expressing the associated set of scaffolding Inc-RNAs together with the required engineered RNAbinding transcriptional regulators (Zalatan et al. 2015). Additionally, the relative expressions of the targeted genes could theoretically be finely modulated through the use of a broad range of transcription factors with different strengths and RNAbinding specificities. This methodology can clearly be applied to the qualitative and quantitative probing of the minimal expression program that is essential for any given cellular phenotype.

Besides reprogramming cells, which can be seen as a variation on the theme of modulating their endogenous expression patterns, the implementation of novel functions has gained significant interest in the life sciences. With potential applications in biotechnology, diagnostics and fundamental research, this approach represents an important aspect of the evolution of synthetic biology in yeast. Many studies have described novel artificial functions and behaviors in yeast cells, which often take advantage of heterologous molecular pathways (e.g. Shou, Ram and Vilar 2007; Win and Smolke 2008; Regot et al. 2011; Youk and Lim 2014; Khakhar et al. 2015). One example is the construction of a cell-to-cell communication system in $S$. cerevisiae (Khakhar et al. 2015). A sender strain was engineered to secrete the plant hormone auxin by integration of a large part of the indole-3-acetamide (IAM) pathway from $A$. tumefaciens: this allows conversion of IAM (added to the culture medium) into auxin (Zhao 2010). The receiving strain was obtained by co-expressing a CRISPR guide RNA specific to a GFP reporter together with a fusion protein consisting of dCas9, an auxin-sensitive degron domain from Arabidopsis (Havens et al. 2012) and a transcriptional activator. In the absence of sender cells, GFP was expressed in the receiving cells. However, in a co-culture of both strains, the receiving cells were sensitive to the presence of auxin secreted by the sender cells, leading to degradation of the fusion protein and reduced expression of the GFP reporter (Khakhar et al. 2015). The exploration of the possibilities of this system is very appealing: its derivation will allow the rational study of population dynamics when mixing different groups of cells that, for instance, respond differently to external 
cues while communicating with each other. Interestingly, as this synthetic mechanism shares some molecular elements with the transcription reprogramming approach presented above, their coupling could represent a unique and powerful way of investigating highly complex population behaviors. This again highlights that synthetic biology in simple organisms such as yeast is poised to make important contributions and appears to be only limited by the imagination of researchers.

Finally, using yeast as a test model for more applied biomedical research is a strategy that parallels and benefits from the development of synthetic biology. While it does not fall into a generic synthetic approach per se, it relies on the same tools. Yeast have been integrated in diagnostic tests (Ishioka et al. 1993) and served as screening platforms for the identification of pharmacological compounds in the field of chemobiology (Bach et al. 2003; Couplan et al. 2011; St Onge et al. 2012). In addition, the investigation of factors involved in human diseases has benefited from understanding the phenotypes associated with their heterologous expression in yeast (Outeiro and Lindquist 2003; Willingham et al. 2003). Therefore, engineering yeast cells beyond their natural functions is a logic that applies to a wide range of research areas.

\section{Perspectives: external control of circuit behavior}

So far, I have discussed engineering artificial systems in yeast cells, focusing mostly on the assembly of genetic circuits that can function autonomously, regardless of whether they sustain any biological function. However, one of the very promising future developments of the bottom-up approaches relies on the external control of endogenous pathways both quantitatively and dynamically: in other words, a synthetic network behavior rather than a synthetic molecular circuit. This aims at artificially imposing the dynamics, spatial organization and strength of an intracellular transduction signal and analyzing the response of single and populations of cells to these novel parameters. This way, instead of correlating a phenotype with an observed regulation in time and space, a more direct cause-consequence relationship can be revealed. For instance, cell cycle progression in fission yeast was externally driven using chemical genetics, in which a non-oscillating minimal synthetic CDK module was made sensitive to a small molecule inhibitor (Coudreuse 
and Nurse 2010). Imposing the timing of the switches in CDK activity by exposing these cells to varying concentrations of the inhibitor allowed for dictating the timing of $\mathrm{S}$ and $\mathrm{M}$ phase onsets, thereby validating the proposed quantitative model of cell cycle control (Fisher and Nurse 1996; Stern and Nurse 1996; Coudreuse and Nurse 2010). Strikingly, this example also highlights the fact that externally modulating the behavior of a process may often rely on the molecular rewiring of the associated regulatory pathway.

The idea of externally imposing how a mechanism operates in various yeast species is facilitated by the development of new technologies. A key driver is the dramatic improvement in DNA sequencing, making the analysis of whole genomes a routine experiment and providing the basis for the design of the controllable modules. Moreover, this approach also strongly benefits from advances in chemical genetics, as well as from recent developments in optogenetics and microfluidics. Again, the robustness and ease of manipulation of yeast cells is an important advantage for the application of these strategies, which will bring the resolution at which we modulate network behavior to the next level. Optogenetics, for instance, provides an unrivaled way to reversibly regulate the subcellular localization of a protein of interest. The integration of a light-responsive dimerization system from plants into $S$. cerevisiae allows very fast switching between binding and dissociation of the PhyB and PIF domains by simply exposing cells to $650 \mathrm{~nm}$ (binding) or 750 $\mathrm{nm}$ (dissociation) light pulses (Yang et al. 2013). This can be used to assess the relevance and function of dynamic changes in the compartmentalization of critical regulatory factors. While these alterations have been observed in a number of processes, their impact often remains unknown and cannot truly be tested through permanently anchoring the proteins outside of their normal domains. In parallel, microfluidic technologies have emerged in the last decade as unique tools for the analysis of single cells and populations, especially when coupled to microscopy (Velve-Casquillas et al. 2010; Sackmann, Fulton and Beebe 2014). They offer an unprecedented degree of dynamic modulation of the extracellular environment while allowing the simultaneous analysis of distinct strains or conditions. The use of microvolumes in microfluidic devices is also particularly suited for applying rapid changes in growth conditions or in the concentration of chemical compounds, making it possible to assess highly dynamic and rapid cellular events. In addition, 
microsystems can be used for an ever growing list of approaches, from analyzing the cellular response to concentration gradients to physically perturbing individual cells to spatially separating single or groups of cells (Park et al. 2006; Paliwal et al. 2007; Terenna et al. 2008; Fehrmann et al. 2013). Regardless of the precise question and read-out of the experiment, it is now possible with these technologies to monitor in real time the consequences of imposing a synthetic behavior on a cellular function. The strength of synthetic biology in yeast relies on the combination of all of these methods, which can be difficult to achieve with the same complexity in higher eukaryotes.

\section{Concluding remarks}

Synthetic biology has its roots in conventional genetic engineering, but with the logic of building and studying systems that can fundamentally differ from their endogenous counterparts or that show no or novel functionalities. The notion of a bottom-up approach is not new, but this strategy has taken time to fully develop in eukaryotic models, mostly as a result of the need for an adapted toolbox. Revolutionary or not, it represents a complementary method to top-down strategies, providing alternative views on cellular functions and allowing for the investigation of questions that remained difficult to tackle. Synthetic biology emerged from the convergence of biology and engineering, but strongly relies on amenable chassis. In eukaryotes, yeasts in all their diversity currently have no equal.

\section{Acknowledgements}

I would like to thank Dr. Pei-Yun Jenny Wu for her insightful feedback on this manuscript. This work was supported by the ERC under the European Union's Seventh Framework Program (FP7/2007-2013 - ERC GA n³10849).

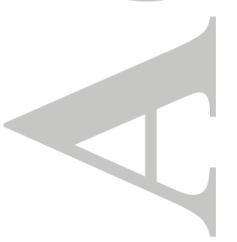




\section{References}

Ajo-Franklin CM, Drubin DA, Eskin JA et al. Rational design of memory in eukaryotic cells. Genes \& development 2007;21:2271-6.

Annaluru N, Muller H, Mitchell LA et al. Total synthesis of a functional designer eukaryotic chromosome. Science 2014;344:55-8.

Bach S, Talarek N, Andrieu T et al. Isolation of drugs active against mammalian prions using a yeast-based screening assay. Nature biotechnology 2003;21:1075-81.

Bashor CJ, Horwitz AA, Peisajovich SG et al. Rewiring cells: synthetic biology as a tool to interrogate the organizational principles of living systems. Annu Rev Biophys 2010;39:515-37.

Blazeck J, Alper H. Systems metabolic engineering: genome-scale models and beyond. Biotechnol J 2010;5:647-59.

Borodina I, Nielsen J. Advances in metabolic engineering of yeast Saccharomyces cerevisiae for production of chemicals. Biotechnol J 2014;9:609-20.

Buijs NA, Siewers V, Nielsen J. Advanced biofuel production by the yeast Saccharomyces cerevisiae. Curr Opin Chem Biol 2013;17:480-8.

Cağatay T, Turcotte M, Elowitz MB et al. Architecture-dependent noise discriminates functionally analogous differentiation circuits. Cell 2009;139:512-22.

Cello J, Paul AV, Wimmer E. Chemical synthesis of poliovirus cDNA: generation of infectious virus in the absence of natural template. Science 2002;297:1016-8.

Chen X, Zhou L, Tian K et al. Metabolic engineering of Escherichia coli: a sustainable industrial platform for bio-based chemical production. Biotechnol Adv 2013;31:1200-23.

Copeland WB, Bartley BA, Chandran D et al. Computational tools for metabolic engineering. Metab Eng 2012;14:270-80.

Coudreuse D, Nurse P. Driving the cell cycle with a minimal CDK control network. Nature 2010;468:1074-9.

Couplan E, Aiyar RS, Kucharczyk R et al. A yeast-based assay identifies drugs active against human mitochondrial disorders. Proc Natl Acad Sci USA 2011;108:11989-94.

Doudna JA, Charpentier E. Genome editing. The new frontier of genome engineering with CRISPR-Cas9. Science 2014;346:1258096-6.

Dymond J, Boeke J. The Saccharomyces cerevisiae SCRaMbLE system and genome minimization. Bioeng Bugs 2012;3:168-71. 
Dymond JS, Richardson SM, Coombes CE et al. Synthetic chromosome arms function in yeast and generate phenotypic diversity by design. Nature 2011;477:471-6.

Elowitz M, Lim WA. Build life to understand it. Nature 2010;468:889-90.

Elowitz MB, Leibler S. A synthetic oscillatory network of transcriptional regulators. Nature 2000;403:335-8.

Fehrmann S, Paoletti C, Goulev $Y$ et al. Aging yeast cells undergo a sharp entry into senescence unrelated to the loss of mitochondrial membrane potential. Cell Rep 2013:5:1589-99.

Fisher DL, Nurse P. A single fission yeast mitotic cyclin B p34cdc2 kinase promotes both S-phase and mitosis in the absence of G1 cyclins. The EMBO journal 1996;15:850-60.

Fitzpatrick J, Kricka W, James TC et al. Expression of three Trichoderma reesei cellulase genes in Saccharomyces pastorianus for the development of a two-step process of hydrolysis and fermentation of cellulose. J Appl Microbiol 2014;117:96-108.

Galanie S, Thodey K, Trenchard IJ et al. Complete biosynthesis of opioids in yeast. Science 2015;349:1095-100.

Gellissen G, Kunze G, Gaillardin C et al. New yeast expression platforms based on methylotrophic Hansenula polymorpha and Pichia pastoris and on dimorphic Arxula adeninivorans and Yarrowia lipolytica - a comparison. FEMS Yeast Res 2005;5:1079-96.

Gérard C, Tyson JJ, Coudreuse D et al. Cell cycle control by a minimal Cdk network. Saucerman JJ (ed.). PLoS computational biology 2015;11:e1004056.

Gibson DG, Glass JI, Lartigue C et al. Creation of a bacterial cell controlled by a chemically synthesized genome. Science 2010;329:52-6.

Goryachev AB, Pokhilko AV. Dynamics of Cdc42 network embodies a Turing-type mechanism of yeast cell polarity. FEBS letters 2008;582:1437-43.

Goto DB, Nakayama J-I. RNA and epigenetic silencing: insight from fission yeast. Dev Growth Differ 2012;54:129-41.

Havens KA, Guseman JM, Jang SS et al. A synthetic approach reveals extensive tunability of auxin signaling. Plant Physiol 2012;160:135-42.

Holoch D, Moazed D. RNA-mediated epigenetic regulation of gene expression. Nat Rev Genet 2015;16:71-84.

Hong K-K, Nielsen J. Metabolic engineering of Saccharomyces cerevisiae: a key cell factory platform for future biorefineries. Cell Mol Life Sci 2012;69:2671-90.

Howell AS, Savage NS, Johnson SA et al. Singularity in polarization: rewiring yeast 
cells to make two buds. Cell 2009;139:731-43.

Hutchison CA, Chuang R-Y, Noskov VN et al. Design and synthesis of a minimal bacterial genome. Science 2016;351:aad6253-3.

Ishioka C, Frebourg T, Yan YX et al. Screening patients for heterozygous p53 mutations using a functional assay in yeast. Nature genetics 1993;5:124-9.

Khakhar A, Bolten NJ, Nemhauser J et al. Cell-Cell Communication in Yeast Using Auxin Biosynthesis and Auxin Responsive CRISPR Transcription Factors. ACS Synth Biol 2015:150706075349008.

Kozubowski L, Saito K, Johnson JM et al. Symmetry-breaking polarization driven by a Cdc42p GEF-PAK complex. Current biology : CB 2008;18:1719-26.

Kricka W, Fitzpatrick J, Bond U. Metabolic engineering of yeasts by heterologous enzyme production for degradation of cellulose and hemicellulose from biomass: a perspective. Front Microbiol 2014;5:174.

Krivoruchko A, Siewers V, Nielsen J. Opportunities for yeast metabolic engineering: Lessons from synthetic biology. Biotechnol J 2011;6:262-76.

Mali P, Esvelt KM, Church GM. Cas9 as a versatile tool for engineering biology. Nat Methods 2013;10:957-63.

Morgan DO. Cyclin-dependent kinases: engines, clocks, and microprocessors. Annu Rev Cell Dev Biol 1997;13:261-91.

Morgan DO. The Cell Cycle. New Science Press, 2007.

Mukherji S, van Oudenaarden A. Synthetic biology: understanding biological design from synthetic circuits. Nat Rev Genet 2009;10:859-71.

Muller $\mathrm{H}$, Annaluru N, Schwerzmann JW et al. Assembling large DNA segments in yeast. Methods in molecular biology 2012;852:133-50.

Naito T, Matsuura A, Ishikawa F. Circular chromosome formation in a fission yeast mutant defective in two ATM homologues. Nature genetics 1998;20:203-6.

Nakamura TM, Cooper JP, Cech TR. Two modes of survival of fission yeast without telomerase. Science 1998;282:493-6.

Nandagopal N, Elowitz MB. Synthetic biology: integrated gene circuits. Science 2011;333:1244-8.

Nevoigt E. Progress in metabolic engineering of Saccharomyces cerevisiae. Microbiol Mol Biol Rev 2008;72:379-412.

Nielsen J, Larsson C, van Maris A et al. Metabolic engineering of yeast for production of fuels and chemicals. Curr Opin Biotechnol 2013;24:398-404.

Ostergaard S, Olsson L, Nielsen J. Metabolic engineering of Saccharomyces 
cerevisiae. Microbiol Mol Biol Rev 2000;64:34-50.

Osterlund T, Nookaew I, Nielsen J. Fifteen years of large scale metabolic modeling of yeast: developments and impacts. Biotechnol Adv 2012;30:979-88.

Outeiro TF, Lindquist S. Yeast cells provide insight into alpha-synuclein biology and pathobiology. Science 2003;302:1772-5.

Paddon CJ, Keasling JD. Semi-synthetic artemisinin: a model for the use of synthetic biology in pharmaceutical development. Nat Rev Microbiol 2014;12:355-67.

Paddon CJ, Westfall PJ, Pitera DJ et al. High-level semi-synthetic production of the potent antimalarial artemisinin. Nature 2013;496:528-32.

Paliwal S, Iglesias PA, Campbell K et al. MAPK-mediated bimodal gene expression and adaptive gradient sensing in yeast. Nature 2007;446:46-51.

Park MC, Hur JY, Kwon KW et al. Pumpless, selective docking of yeast cells inside a microfluidic channel induced by receding meniscus. Lab Chip 2006;6:988-94.

Pósfai G, Plunkett G, Fehér T et al. Emergent properties of reduced-genome Escherichia coli. Science 2006;312:1044-6.

Rabinovitch-Deere CA, Oliver JWK, Rodriguez GM et al. Synthetic biology and metabolic engineering approaches to produce biofuels. Chem Rev 2013;113:4611-32.

Regot S, Macia J, Conde N et al. Distributed biological computation with multicellular engineered networks. Nature 2011;469:207-11.

Ro D-K, Paradise EM, Ouellet $\mathrm{M}$ et al. Production of the antimalarial drug precursor artemisinic acid in engineered yeast. Nature 2006;440:940-3.

Sackmann EK, Fulton AL, Beebe DJ. The present and future role of microfluidics in biomedical research. Nature 2014;507:181-9.

Shen $Y$, Stracquadanio G, Wang $Y$ et al. SCRaMbLE generates designed combinatorial stochastic diversity in synthetic chromosomes. Genome Res 2015;26:36-49.

Shou W, Ram S, Vilar JMG. Synthetic cooperation in engineered yeast populations. Proc Natl Acad Sci USA 2007;104:1877-82.

Sprinzak D, Elowitz MB. Reconstruction of genetic circuits. Nature 2005;438:443-8.

St Onge R, Schlecht U, Scharfe $\mathrm{C}$ et al. Forward chemical genetics in yeast for discovery of chemical probes targeting metabolism. Molecules 2012;17:13098115.

Stern B, Nurse P. A quantitative model for the cdc2 control of $S$ phase and mitosis in fission yeast. Trends Genet 1996;12:345-50. 
Sternberg SH, Doudna JA. Expanding the Biologist's Toolkit with CRISPR-Cas9. Molecular cell 2015;58:568-74.

Stricker J, Cookson S, Bennett MR et al. A fast, robust and tunable synthetic gene oscillator. Nature 2008;456:516-9.

Szczebara FM, Chandelier C, Villeret C et al. Total biosynthesis of hydrocortisone from a simple carbon source in yeast. Nature biotechnology 2003;21:143-9.

Takahashi S, Pryciak PM. Membrane localization of scaffold proteins promotes graded signaling in the yeast MAP kinase cascade. Current biology : $C B$ 2008;18:1184-91.

Tao EY, Calvert M, Balasubramanian MK. Rewiring Mid1p-independent medial division in fission yeast. Current biology : CB 2014;24:2181-8.

Terenna CR, Makushok T, Velve-Casquillas G et al. Physical mechanisms redirecting cell polarity and cell shape in fission yeast. Current biology : $C B$ 2008;18:1748-53.

Velve-Casquillas G, Le Berre M, Piel M et al. Microfluidic tools for cell biological research. Nano Today 2010;5:28-47.

Wiechert W. Modeling and simulation: tools for metabolic engineering. Journal of biotechnology 2002;94:37-63.

Willingham S, Outeiro TF, DeVit MJ et al. Yeast genes that enhance the toxicity of a mutant huntingtin fragment or alpha-synuclein. Science 2003;302:1769-72.

Win MN, Smolke CD. Higher-order cellular information processing with synthetic RNA devices. Science 2008;322:456-60.

Yang X, Jost AP-T, Weiner OD et al. A light-inducible organelle-targeting system for dynamically activating and inactivating signaling in budding yeast. Molecular biology of the cell 2013;24:2419-30.

Youk H, Lim WA. Secreting and sensing the same molecule allows cells to achieve versatile social behaviors. Science 2014;343:1242782-2.

Zalatan JG, Lee ME, Almeida $R$ et al. Engineering complex synthetic transcriptional programs with CRISPR RNA scaffolds. Cell 2015;160:339-50.

Zhao Y. Auxin biosynthesis and its role in plant development. Annu Rev Plant Biol 2010;61:49-64.

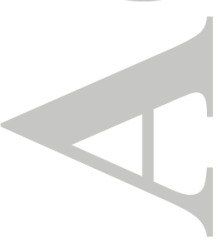




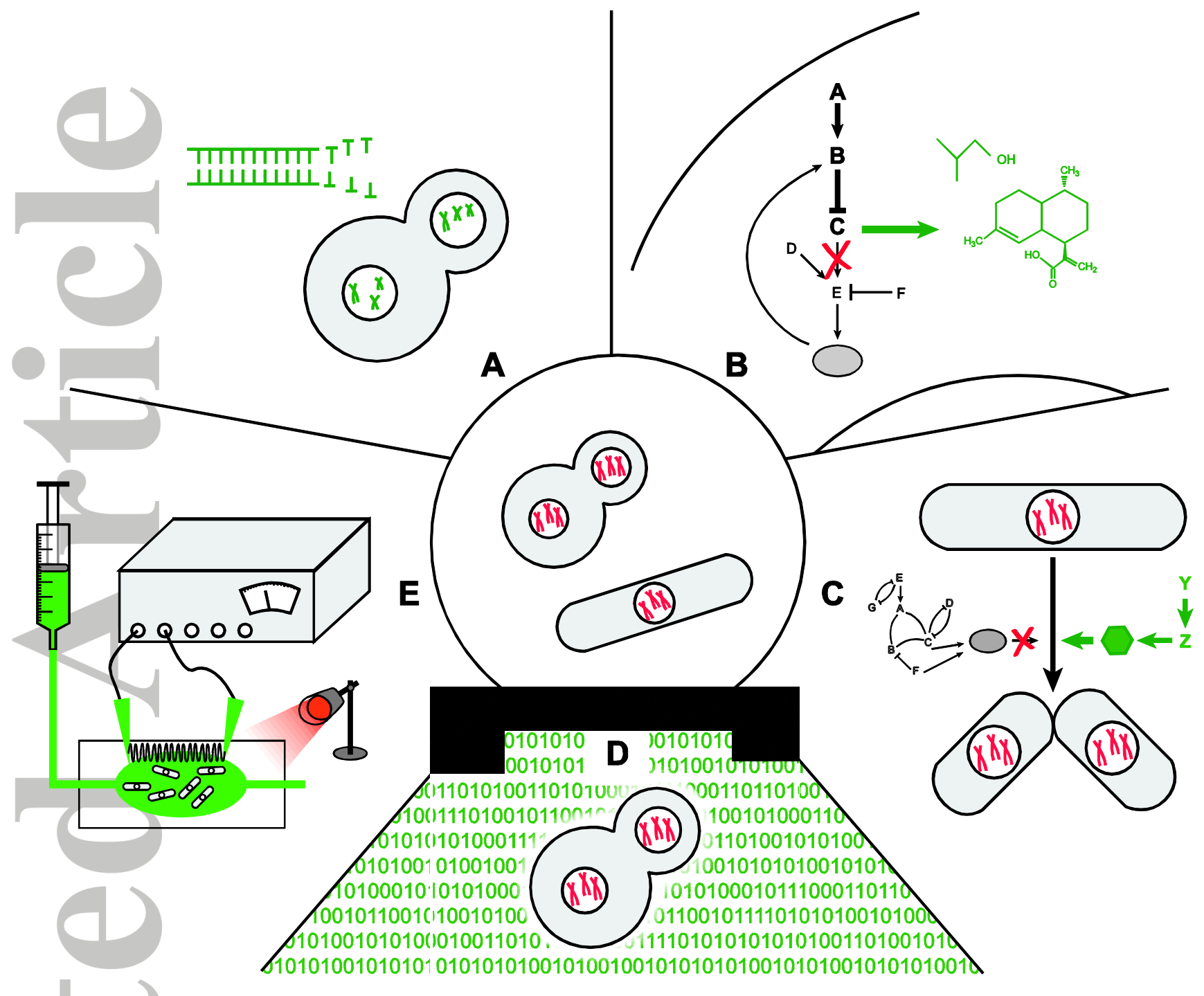

Figure 1.lllustration of the different aspects of synthetic biology in yeast, as discussed in this review. A. Genome engineering. B. Hijacking molecular pathways. C. Recapitulating cellular functions. D. From proof-of-concept circuits to novel programs and functions. E. External control of circuit behavior. 


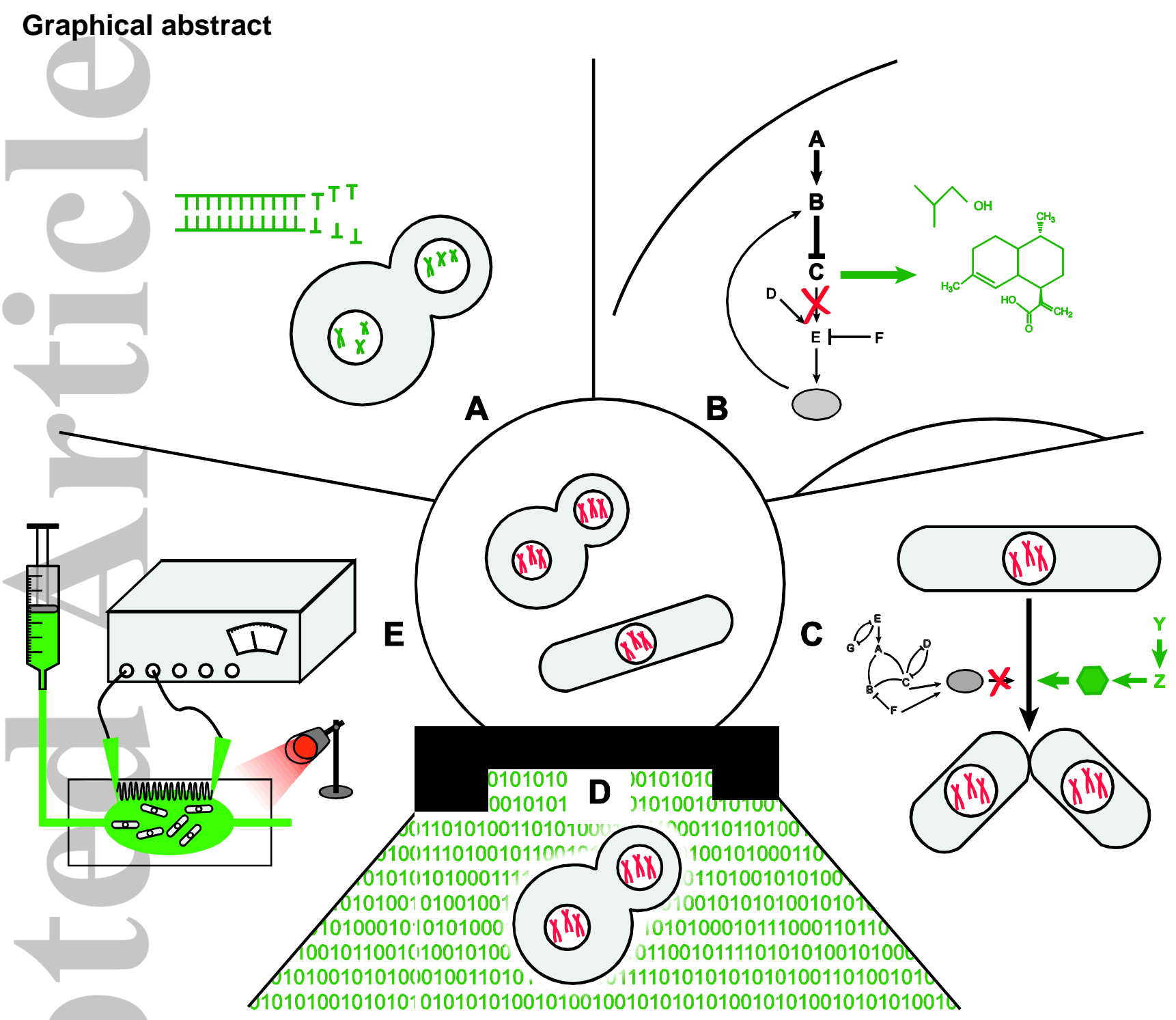

Synthetic biology has made a remarkable entry into the life sciences, playing an important role in our understanding of biological processes. From engineering novel genomes to transforming cells into chemical factories, yeasts represent ideal chassis for the application and future development of this bottom-up strategy in eukaryotes. 